\title{
A IOT (INTERNET OF THINGS) NO CENÁRIO BRASILEIRO: SUAS VANTAGENS E OS PRINCIPAIS DESAFIOS PARA SUA IMPLEMENTAÇÃO E EXPANSÃO
}

\author{
Ciro Ávila Machado Ferreira - ciro.ferreira@alunos.unis.edu.br \\ Guilherme Reis Portilho - guilherme.portilho@alunos.unis.edu.br \\ Rodolfo Martins Albuini - rodolfo.albuini@alunos.unis.edu.br \\ Victor Hugo Silva Lucas - victor.lucas@ alunos.unis.edu.br \\ Prof ${ }^{\mathrm{D}}$ Orientador: Sanderson Rocha de Abreu - sanderson.abreu@unis.edu.br
}

\begin{abstract}
RESUMO
A Internet das Coisas (IoT - Internet of Things) consiste na inserção de objetos do cotidiano à Internet, recebendo uma capacidade computacional de comunicação. Dessa forma, eles passam a receber e enviar dados e adquirem novas funcionalidades quando interconectados à rede mundial. Diante de seus inúmeros benefícios, como a criação de um banco de dados dos perfis dos consumidores ou a automação de qualquer processo ou tarefa, tornando-os mais eficientes e seguros, a IoT possui aplicabilidade para diferentes setores tanto econômicos quantos sociais, como o comércio, as indústrias, a saúde, a agricultura e até mesmo no conceito de cidade inteligente. Nesse contexto, os autores desse artigo se propuseram a analisar, compreender e apresentar uma visão dos conceitos de indústria 4.0, de IoT e das principais dificuldades enfrentadas na implementação dessa nova tecnologia, principalmente, no cenário brasileiro.
\end{abstract}

Palavras - chaves: Internet das Coisas; Indústria 4.0; Inovação; Tecnologia; IoT no Brasil.

\begin{abstract}
The Internet of Things (IoT - Internet of Things) consists of the insertion of everyday objects into the Internet, receiving a computational communication capacity. In this way, they start to receive and send data and acquire new features when interconnected to the global network. In view of its numerous benefits, such as the creation of a database of consumer profiles or the automation of any process or task, making them more efficient and safe, IoT has applicability for different sectors, both economic and social, such as commerce, industries, health, agriculture and even the concept of smart city. In this context, the authors of this article set out to analyze, understand and present a view of the concepts of industry 4.0, IoT and the main difficulties faced in the implementation of this new technology, mainly in the Brazilian scenario.
\end{abstract}

Keys: Internet of Things; Industry 4.0; Innovation; Technology; IoT in the Brazil.

\section{INTRODUÇÃO}

Com o avanço da tecnologia, novas demandas surgiram tanto por parte das indústrias quanto do mercado consumidor. Esse novo cenário fomentou a produção de novos conhecimentos baseados, principalmente, na inovação e na era digital. Segundo SCHWAB (2016), o mundo vive no prelúdio de uma quarta revolução industrial, determinada pelo uso intensivo de tecnologias digitais a fim de fabricar novos produtos de forma rápida, atendendo as demandas de forma ágil e otimizando em tempo real a produção e a cadeia de suprimentos. 
Dentre essas tecnologias que fazem e farão parte da nova era da indústria, destaca-se a IoT (Internet das Coisas ou do termo em inglês Internet Of Things).

A IoT consiste na inserção de objetos do cotidiano à internet, recebendo uma capacidade computacional e de comunicação. Dessa forma, eles passam a receber e enviar dados pela rede mundial de computadores (SANTOS et al., 2017). Essa conjuntura permite caracteriza-la como o coração da Indústria 4.0, pois, permite uma união mais intensa entre as máquinas virtuais e físicas, remodelando e otimizando toda a cadeia de produção desde os fornecedores até os clientes finais (SOLDATOS et al., 2016).

Do ponto de vista econômico e de gestão, o termo a Internet das Coisas foi usado pela primeira vez por Elggar Fleisch em 2010. De acordo com FLEISCH (2010), apesar do conceito não ser novo, apenas recentemente tornou-se relevante do ponto de vista prático nos negócios, em razão dos avanços no desenvolvimento de hardware, em especial na última década. Dados apontam um crescimento notório nas parcelas da economia interligadas aos dispositivos de IoT, segundo GARTNER (2013), os segmentos que liderarão a adoção dessa tecnologia serão a manufatura (15\%), a saúde (15\%) e os seguros $(11 \%)$.

Considerando a relevância do tema, o presente artigo possui como objetivo geral revisar a literatura com o intuito de entender o conceito de Indústria 4.0 e o conceito, surgimento e história da Internet das Coisas. O objetivo principal é buscar identificar as vantagens dessa tecnologia para a nova era da indústria e para a sociedade, além de apresentar as principais dificuldades da sua implementação e expansão no cenário brasileiro.

O artigo se propõe a responder questões como: O que é IoT? Qual a sua importância no cenário mundial e brasileiro? Qual sua relevância para a indústria e para a sociedade? Quais barreiras são enfrentadas para sua implementação e expansão? Através da pesquisa pelas respostas dessas indagações, esse trabalho contribui, ainda que ínfimo, para o avanço e estímulo dos estudos nessa área pouco explorada até o momento.

\section{METODOLOGIA}

A escolha da metodologia torna-se fundamental no direcionamento de uma pesquisa. Para a elaboração desse artigo, utilizou-se de uma análise de referencial teórico que é desenvolvida a partir do estudo de materiais previamente elaborados, principalmente livros e artigos científicos (GIL, 1991).

Segundo Lakatos e Marconi (2003), o referencial teórico também permite averiguar o estado do problema a ser pesquisado sob a ótica da teoria. Dessa maneira, conforme pontuado por Marion et al. (2002), esse modelo de metodologia deve conter uma síntese do que existe de 
mais atual na abordagem do tema definido ainda que as teorias atuais não façam parte das escolhas do pesquisador.

O objetivo desse artigo é analisar, compreender e apresentar uma visão geral dos conceitos de indústria 4.0 e de Internet das Coisas (Internet of Things), considerando os aspectos técnicos, e apresentar informações sobre os seus benefícios para a sociedade e as dificuldades enfrentadas para a implementação e expansão dessa nova tecnologia, principalmente, no cenário brasileiro.

\section{DESENVOLVIMENTO}

\subsection{Indústria 4.0}

Desde a primeira revolução industrial, a indústria visa melhorar sua performance através da busca de soluções inovadoras. Essa postura impulsiona o desenvolvimento de diversas tecnologias em todas as áreas interligadas à produção (SUGAYAMA; NEGRELLI, 2015), conforme pode ser observado no Quadro I.

\begin{tabular}{|c|l|l|}
\hline $\begin{array}{c}\text { Revolução } \\
\text { Industrial }\end{array}$ & \multicolumn{1}{|c|}{ Período } & \multicolumn{1}{c|}{ Tecnologias desenvolvidas } \\
\hline $\begin{array}{c}\text { Primeira } \\
\text { Revolução } \\
\text { Industrial }\end{array}$ & $\begin{array}{l}\text { Iniciou na segunda metade } \\
\text { do século XVIII. Ocorreu } \\
\text { entre 1760 e 1840. }\end{array}$ & $\begin{array}{l}\text { Introdução da máquina a vapor, usando a água e o vapor para } \\
\text { mecanizar a produção que antes era essencialmente artesanal. }\end{array}$ \\
\hline $\begin{array}{c}\text { Segunda } \\
\text { Revolução } \\
\text { Industrial }\end{array}$ & $\begin{array}{l}\text { Iniciou no século XIX e } \\
\text { avançou a primeira metade } \\
\text { do século XX. }\end{array}$ & $\begin{array}{l}\text { Advento da energia elétrica facilitando as linhas de produção e } \\
\text { a produção em massa. }\end{array}$ \\
\hline $\begin{array}{c}\text { Terceira } \\
\text { Revolução }\end{array}$ & $\begin{array}{l}\text { Iniciou na década de 1960 } \\
\text { (segunda metade do século } \\
\text { XX) e avançou até a } \\
\text { década de 1990. }\end{array}$ & $\begin{array}{l}\text { Implementação de componentes eletrônicos e tecnologia que } \\
\text { permitiram a automação dos processos produtivos. }\end{array}$ \\
\hline $\begin{array}{c}\text { Quarta } \\
\text { Revolução } \\
\text { Industrial }\end{array}$ & $\begin{array}{l}\text { Iniciou na primeira década } \\
\text { do século XXI, na década } \\
\text { de 2000. }\end{array}$ & $\begin{array}{l}\text { Digitalização da produção, possibilitando a personalização da } \\
\text { produção em massa caracterizada pela internet ubíqua e móvel, } \\
\text { sensores menores e mais poderosos e a inteligência artificial, } \\
\text { com mudanças profundas na forma de produção e de consumo, } \\
\text { desencadeando o desenvolvimento de novos modelos de } \\
\text { negócios. }\end{array}$ \\
\hline
\end{tabular}

Quadro 1. Desenvolvido pelos autores.

O conceito Indústria 4.0 surgiu pela primeira vez em 2001, durante a feira Hannover Messe, sediada na Alemanha, despertando o interesse acadêmico, científico, empresarial e político sobre o tema. $\mathrm{O}$ desenvolvimento de pesquisas tem se expandido rapidamente e alguns autores apostam no fato de que pela primeira vez uma revolução industrial está sendo observada antes de se tornar, concretamente, realidade (HERMANN; PENTEK; OTTO, 2015). 
Essa nova era industrial é encarada como estratégica pelas grandes potências industriais. Por isso, algumas empresas passaram a desenvolver soluções para esse novo conceito, apoiadas por governos, principalmente europeus, e por países como Estados Unidos, Japão e China (SUGAYAMA; NEGRELLI, 2015).

Segundo Coelho (2016), a indústria está estreitamente ligada ao processo de melhoria contínua em termos de eficiência, segurança, produtividade das operações e, especialmente, no retorno do investimento. São várias as tecnologias e tendências facilitadoras disponíveis, porém, pode-se destacar as seguintes tecnologias como os pilares dessa nova era: IoT - Internet das Coisas, Computação em Nuvem, Big Data and Analytics, Realidades Virtual e Aumentada, Cyber-Physical Systems (Sistemas Ciber-físicos) e Impressão 3D.

Tendo em visto a importância da $4^{\mathrm{a}}$ Revolução Industrial e da IoT dentro dessa nova realidade, o presente artigo se detém a explicar sobre essa tecnologia e pontuar os principais problemas enfrentados para a sua implementação e expansão - tanto no cenário brasileiro quanto mundial.

\subsection{O que é IoT?}

Segundo Zaslavsky et al. (2013), o termo Internet das Coisas - Internet of Things (IoT) surgiu em meados de 1999 a partir de ideias do pesquisador britânico Kevin Ashton do MIT (Instituto de Tecnologia de Massachusetts). Nessa época, o pesquisador identificou a necessidade de etiquetar eletronicamente os produtos da linha de produção de uma empresa através de identificadores de radiofrequência, visando facilitar a logística. Essa visão se expandiu e notou-se a possibilidade de uma interligação autônoma direta entre dispositivos, proporcionando a automação de processos tanto simples quanto complexos, facilitando a vida do ser humano.

Apesar dessa percepção, a primeira definição de Internet das Coisas - Internet of Things (IoT) surge em 2005 em um relatório da ITU (International Telecommunications Union) das Nações Unidas. Nesse relatório, a IoT foi definida como a conexão de todos os objetos e dispositivos do cotidiano a todos os tipos de redes: intranets, redes peer-to-peer e a internet global (ITU, 2005). A ideia básica da IoT é que, virtualmente, todas as coisas físicas no mundo podem adquirir algumas características de pequenos computadores e tornarem-se objetos inteligentes (FLEISH, 2010).

A IoT é a combinação de diferentes tecnologias de desenvolvimento que se complementam e fornecem capacidades para auxiliar no preenchimento da lacuna existente entre o mundo virtual e físico. Dentre essas capacidades, pode-se destacar: comunicação e 
cooperação, endereçamento, identificação, detecção do ambiente, ação, processamento de informação embarcada, localização e interface com o usuário (MATTERN E FLOERKEMEIER, 2010).

Segundo Fleisch (2010), devido a abrangência do tema, o campo de pesquisa sobre IoT passa por diversas áreas de conhecimento, como: computação, engenharias, telecomunicações, design, economia e negócios. Portanto, com base na literatura pesquisada e procurando abranger o máximo possível desse campo de pesquisa, o quadro 2 relaciona a definição de IoT de acordo com autores de diferentes áreas.

\begin{tabular}{|c|c|c|}
\hline Autores & Definição & Área de conhecimento \\
\hline ITU (2005) & $\begin{array}{l}\text { Internet das Coisas engloba a conexão de } \\
\text { objetos e dispositivos do cotidiano em todos os } \\
\text { tipos de redes, por exemplo: intranets, redes } \\
\text { peer-to-peer e a internet global. }\end{array}$ & Telecomunicações \\
\hline FLEISCH (2010) & $\begin{array}{l}\text { Todas as coisas físicas no mundo podem se } \\
\text { tornar computadores que se conectam à Internet, } \\
\text { ou seja, as coisas passam a ter algumas } \\
\text { características de pequenos computadores, } \\
\text { tornam-se, então, objetos inteligentes. }\end{array}$ & Administração e Economia \\
\hline $\begin{array}{l}\text { MATTERN E } \\
\text { FLOERKEMEIER } \\
\qquad(\mathbf{2 0 1 0})\end{array}$ & $\begin{array}{l}\text { Internet das Coisas representa uma visão } \\
\text { segundo a qual a internet se estende ao mundo } \\
\text { real por meio de objetos do cotidiano. }\end{array}$ & Computação pervasiva \\
\hline $\begin{array}{c}\text { TAN E WANG } \\
(\mathbf{2 0 1 0})\end{array}$ & $\begin{array}{l}\text { Internet das Coisas será a próxima geração da } \\
\text { internet, em que todos os objetos estarão } \\
\text { conectados. Representa uma nova era da } \\
\text { computação ubíqua. }\end{array}$ & Computação ubíqua \\
\hline$\underset{(2010)}{\text { ATZORI ET AL. }}$ & $\begin{array}{l}\text { Internet das Coisas é um novo paradigma que } \\
\text { consiste na presença pervasiva dos objetos e } \\
\text { "coisas" inteligentes ao nosso redor - tais como } \\
\text { RFID tags, sensores, actuators, telefones } \\
\text { móveis -, os quais estarão prontos para interagir } \\
\text { e cooperar uns com os outros a fim de atingir } \\
\text { um objetivo específico. }\end{array}$ & Computação - Rede de computadores \\
\hline $\begin{array}{l}\text { KORESHOFF, } \\
\text { ROBERTSON E } \\
\text { LEONG (2013) }\end{array}$ & $\begin{array}{l}\text { Internet das Coisas se refere a uma visão mais } \\
\text { ampla, na qual "coisas" são objetos, lugares, } \\
\text { ambientes do cotidiano. Todas essas "coisas" } \\
\text { estão interconectadas umas às outras pela } \\
\text { internet. }\end{array}$ & Interação Homem-Computador \\
\hline
\end{tabular}

Quadro 2. Desenvolvido pelos autores.

Baseando nas informações supracitada, resumidamente, na IoT as "coisas" ou objetos devem participar ativamente em processos de negócios, em processos informacionais e sociais, possibilitando comunicações inter-relacionais, com o propósito de cruzar as informações 
coletadas do ambiente, reagindo de forma autônoma aos eventos do mundo físico e real sem intervenção direta humana.

\subsection{Vantagens e aplicações para IoT}

A Internet tornou-se parte fundamental da sociedade. No futuro, estima-se que a rede e os computadores estarão tão integrados a rotina das pessoas, disfarçados nos objetos do cotidiano, que serão imperceptíveis (MOREIRAS, 2014). Equipamentos como eletrodomésticos, meios de transporte, roupas e maçanetas estarão conectados à Internet e a outros dispositivos, como computadores e smartphones, ou seja, nota-se uma revolução tecnológica desencadeada pela Internet das Coisas (ZAMBARDA, 2014).

Portanto, diante da sua importância, o Conselho Nacional de Inteligência dos EUA (NIC) considera a IoT como uma das seis tecnologias civis mais promissoras e de maior impacto a nação no futuro próximo. A previsão é de que em 2025 todos os objetos do cotidiano estarão conectados à Internet.

Em vista disso, embasado nas pesquisas realizadas foi possível detectar as seguintes aplicações para IoT:

\section{a) Cidades Inteligentes}

Segundo Zanella et al. (2014), uma aplicação para cidades inteligentes seria na coleta de resíduos. Segundo o autor, é possível otimizar o percurso dos caminhões coletores através da utilização de recipientes de resíduos inteligentes com a função de detectar o nível de carga, o que pode levar a uma redução nos custos ao recolher os resíduos e ainda melhorar a qualidade da reciclagem. Para realizar tal serviço, a IoT deve conectar os recipientes inteligentes a um centro de controle, onde através de um software de otimização ocorrerá o processamento dos dados e o gerenciamento ideal da coleta da frota de caminhões.

Outro exemplo importante, segundo Sannapureddy (2015), consiste na detecção da poluiçãa e de calamidades naturais. O uso da IoT permitiria monitorar as emissões de gases poluentes, acompanhar a liberação de produtos químicos e resíduos nocivos nos rios e no mar, prevenindo e/ou minimizando tanto a poluição do ar quanto da água. Além dessas situações, ainda é possível emitir alertas de terremotos e tsunamis, por exemplo, através da detecção de tremores.

\section{b) Logística}

De acordo com Bassi et al. (2013), em relação a área de logística de transporte, a IoT proporciona tanto melhorias nos sistemas de fluxos de materiais quanto influencia no 
posicionamento global e identificação automática de mercadorias. Ademais, proporciona uma diminuição no consumo de energia através do aumento da eficiência energética. Com isso, os autores concluem que é esperada grandes mudanças na cadeia global de suprimentos por meio de movimentação de carga inteligente. Para alcançar esse objetivo, é necessário a sincronização contínua de informações da cadeia em tempo real, tornando a cadeia mais transparente, confiável e controlável.

\section{c) Agricultura}

A tecnologia IoT é capaz de gerar um grande potencial para melhorar a segurança e a qualidade dos produtos agrícolas. Através do monitoramento contínuo do plantio e da colheita, é possível uma maior visibilidade dos estágios de produção por parte dos produtores, o que promove um controle mais apurado da produção (LIU et al., 2015).

\section{d) Saúde}

No campo da saúde, também haverá aplicações. De acordo com Domingo (2012), pode-se citar como exemplo os casos de pacientes paralisados que utilizam fraldas. Um sensor de umidade poderia alertar aos enfermeiros e cuidadores para substituir a fralda imediatamente após a detecção de umidade no nível máximo de absorção suportado pela estrutura da fralda.

\subsection{Desafios técnicos e estruturais enfrentados pela IoT}

Conforme novas aplicações estimulam desafios de conectar objetos à Internet, consequentemente, aumenta a preocupação em manter a segurança e privacidade desses dados. Naturalmente, esses objetos heterogêneos apresentam limitações quanto a capacidade energética, hardware e comunicação. Sendo assim, certas questões são exploradas a fim de promover o endereçamento de todos esses dispositivos com a manutenção da melhor taxa de entrega e com baixo uso dos recursos.

Segundo Loes (2015), é impossível conter o movimento no sentido de adoção maciça da IoT, entretanto, muitos desafios serão enfrentados durante o processo de implantação dessa tecnologia, como armazenamento de dados, segurança das informações, funcionários bem capacitados, entre outros, que serão vistos abaixo.

\section{a) IPv4}

Para um correto funcionamento da Internet, são necessários protocolos de endereçamento e encaminhamentos de pacotes, entre outras funções, sendo o IP um dos protocolos mais importantes. A versão mais utilizada é a 4, conhecida como IPv4, que possui 
32 bits de endereçamento, o que possibilita quatro bilhões de endereços disponíveis aproximadamente. Todavia, esses IPs esgotaram-se em decorrência do grande número de dispositivos conectados.

\section{b) IPv6}

Com o objetivo de solucionar o problema supracitado e visando o contínuo crescimento da rede, foi desenvolvido o IPv6. Segundo Pierini (2014), esse protocolo atenderá por bastante tempo as necessidades da Internet, entretanto, existe uma incompatibilidade entre os dois protocolos, o que resulta em uma migração complexa e de custo elevado.

\section{c) Armazenamento das informações}

Gubbi et al. (2013) destacam que os sistemas, os servidores de armazenamentos e de redes precisam se desenvolver para dar suporte a transição e o crescimento maciço dos novos dispositivos. A realidade da IoT irá estabelecer novas exigências em armazenamento e, em contrapartida, esses dados precisarão ser armazenados em um lugar seguro para serem úteis posteriormente.

\section{d) Infraestrutura}

Através da IoT será possível a criação de uma nova geração de ecossistemas dinâmicos em ambientes como cidades inteligentes e hospitais. Entretanto, ecossistemas dinâmicos demandam acesso ubíquo à Internet, transferência de dados sem descontinuidade, políticas de roaming flexíveis e um protocolo de mobilidade interoperável com infraestrutura de Internet existente. Essas exigências são desafios enfrentados pelos dispositivos de IoT, uma vez que, geralmente, são dispositivos com pouca capacidade de memória, processamento e comunicação, e de energia limitada (JARA et al., 2014).

Além dos obstáculos supracitados, segundo o CEO da Ericsson, Hans Vestberg, em entrevista à revista Exame (2014), um outro desafio será a interconexão entre operadoras e empresas. De acordo com ele, no ambiente da IoT, diferentes setores precisão se relacionar, não sendo mais apenas uma conversa entre operadores de telecomunicações e seus fornecedores.

\section{e) Segurança e Privacidade das informações}

De acordo com Chen et al. (2014), entre as redes tradicionais e as redes da IoT, as questões de segurança e privacidade tornam-se mais relevantes e preocupantes no segundo ambiente. A proteção da privacidade será um importante ponto a ser considerado, visto que o fluxo de dados entre os dispositivos inclui informações confidenciais e particulares. 
Diante do alto grau de conectividade entre dois ou mais dispositivos e com um intenso tráfego de informações, a IoT abre portas para uma série de riscos aos usuários, tanto domésticos quanto empresariais. Essas ameaças podem ser oriundas dos próprios dispositivos ou das plataformas e redes nos quais estão inseridos (ABREU, 2016). Portanto, a segurança será uma das principais preocupações em locais onde forem implantadas redes em larga escala. Existem muitas maneiras do sistema ser atacado, como desativando a disponibilidade da rede, empurrando dados errôneos para a rede, acesso à informação pessoal, entre outros (GUBBI et al., 2013).

Segundo Atzori et. Al (2010), a IoT é vulnerável a ataques por várias razões, entre elas pode-se destacar a ausência de vigilância dos seus componentes, tornando-os alvos fáceis para ataques físicos, e a comunicação sem fio, tornando a espionagem mais simples. Por isso, segundo o autor, mecanismos comumente utilizados como criptografia leve, protocolos de segurança e garantia da privacidade, não são mais suficientes para a IoT. Os mecanismos dos protocolos de segurança devem ser analisados e avaliados se estão aptos a serem integrados nessa nova tecnologia, considerando a menor capacidade de processamento, memória e energia dos dispositivos IoT.

\section{f) Mão de obra especializada}

Segundo Bassi et al. (2013), haverá uma redução no número de funcionários necessários devido a automatização dos procedimentos ocasionada pela IoT. Algumas funções serão substituídas por máquinas como scanners de código de barras, leitores, sensores, atuadores e até mesmo complexos robôs tão eficientes quanto um ser humano. Entretanto, essas tecnologias provocarão uma necessidade de profissionais qualificados para programar e reparar os equipamentos e sensores.

Acompanhando essa mesma linha de raciocínio, pode-se inferir que haverá uma geração de novos empregos com oportunidades para desenvolvedores de software e para engenheiros de telecomunicações, por exemplo. Os primeiros terão um universo novo para trabalhar enquanto os segundos serão os responsáveis pelo desenvolvimento e pela manutenção de toda infraestrutura de rede e segurança.

\section{g) Interoperabilidade}

Segundo Silva (2004), interoperabilidade é a capacidade de um sistema (informatizado ou não) se comunicar de forma transparente com outro sistema - semelhante ou não. É muito importante que o sistema trabalhe com padrões abertos. Entretanto, segundo o autor, o mercado 
caminha cada vez mais para a criação de padrões para sistemas, tornando esse tema um grande desafio para a instauração da IoT.

O teste de interoperabilidade é uma ferramenta que acelera o desenvolvimento e possibilita a avaliação de desempenho por outros fabricantes e/ou desenvolvedores que não sabem como foi desenvolvida cada solução. Além disso, é possível testar se os padrões estabelecidos estão sendo utilizados corretamente e se aquela solução/produto consegue trabalhar corretamente dentro dos limites das normas (MARÃO, 2013).

\section{h) Sustentabilidade}

Analisando um futuro próximo, percebe-se a existência de desafios envolvendo questões energéticas e o desenvolvimento de sistemas eficientes de energia e autossustentáveis, sendo imprescindíveis ao suporte de aplicações em IoT. Conforme Chen et al. (2014), devem ser desenvolvidas novas formas de energia ambientais e ampliadas a eficiência no processamento e na comunicação através de novos circuitos, novos paradigmas de programações e no desenvolvimento de energia eficiente e de protocolos de antenas inteligentes. Como fatores chave para o desenvolvimento de sistemas autônomos inteligentes sem fio podem-se destacar o amadurecimento de novas, eficazes e compactas baterias e uma nova geração de dispositivos com métodos sustentáveis de transmissão ou captação de energia.

\subsection{Cenário brasileiro}

Apesar de ser uma das maiores tendências no mercado mundial, a IoT está chegando no Brasil ainda de forma tímida devido aos grandes desafios que enfrenta para sua implantação no país. De acordo com Guerra (2014), a maior dificuldade hoje é a inexistência de antenas e de espectro de frequência suficientes para endereçar o grande volume de dados que aumentam à medida que se expande a quantidade de dispositivos conectados à rede.

Apesar de algumas empresas privadas brasileiras estarem sendo pioneiras no tema IoT no Brasil, o país ainda não se encontra em um patamar propício a implantação dessa tecnologia no que diz respeito a situação de infraestrutura de telecomunicações. O acesso de tantos dispositivos à rede IoT demanda serviços de acesso móvel a internet com o 5G, que ainda está sendo desenvolvido e testado.

Outras questões necessitam uma atenção especial a fim de proporcionar o desenvolvimento de um campo apropriado a implantação da IoT, como: a falta de cabeamento estruturado e equipamentos que suportarão a demanda de informações, além da dificuldade de 
atender todas as localidades com conexão à internet de alta velocidade em razão das grandes extensões territoriais do país (SANTANA, 2015).

Por fim, outro aspecto negativo está relacionado às iniciativas de empresas e operadoras pontuais, como a regulamentação e a predisposição da indústria de investir e apostar em um novo modelo de negócios (FERRAZ, 2014). Além disso, ainda se enfrenta desafios adicionais, como: a alta carga tributária e um menor poder aquisitivo da população; consumidor menos propenso à experimentação; e a menor disponibilidade de capital de risco e funding com consequente escassez de projetos em iniciativas de IoT (TRAVASSOS, 2015).

\subsection{Visão de futuro para IoT no Brasil}

Diante dos pontos negativos citados anteriormente, ainda não é possível afirmar que a IoT trará toda a acessibilidade e facilidade que se prevê com ela. Comparado aos países desenvolvidos, o Brasil ainda é um país pouco desenvolvido tecnologicamente, dificultando um pouco a implementação e expansão da IoT em sua capacidade máxima.

Apesar desse panorama, Santana (2015) afirma que a IoT pode gerar muitos benefícios para o país desde que seja realizado um trabalho sério por parte dos governantes. Ainda de acordo com o autor, será necessário o amadurecimento de uma relação entre diferentes áreas do conhecimento, como: TI (tecnologia da informação), medicina, engenharia e agricultura, com o objetivo de atingir o valor que a IoT pode trazer para a humanidade. Ele reforça que esse novo paradigma proporcionará novas oportunidades para os brasileiros com a formação de novas empresas atuando direta e indiretamente nesse ramo, além de gerar melhorias na segurança da informação, em infraestrutura e avanços econômicos.

Reforçando essa opinião, Milagres (2015) destaca que as mudanças terão forte aspecto econômico em razão da maior automatização das fábricas, indústrias e comércio. Por um lado, provocando benefícios e melhorias, mas por outro gerando desempregos. Complementando essa ideia, Teotônio (2015) acredita que com a IoT haverá um aumento no PIB nacional em virtude da criação de novos produtos e do investimento em tecnologias. Inicialmente, os benefícios dessa nova tecnologia impactarão uma pequena parcela da população, principalmente, os empreendedores da área de tecnologia. Por esse motivo, os profissionais da área de TI serão a peça chave da fase de implementação da IoT. Além disso, pode-se destacar essa nova como uma oportunidade para o setor de marketing, possibilitando a criação de um banco de dados contendo informações sobre as pessoas para posteriormente traçar um perfil comportamental dos consumidores. 


\section{CONSIDERAÇÕES FINAIS}

Diante a evolução técnica existente, é possível acreditar no potencial da implementação da IoT - conectando todos os dispositivos através da Internet, facilitando a troca de informações e a realização de tarefas envolvendo o mínimo possível de fator humano, tornando-as mais rápidas, seguras e automatizadas. Autores como Asensio et al. (2014) e Jara et al. (2014), acreditam que a IoT será um caminho sem volta assim como a própria Internet se fortaleceu quando computadores ao redor do mundo passaram a ficar conectados.

Apesar de ser um assunto que ganhou maior visibilidade nos últimos anos, pode-se observar que ainda existem barreiras, desafios e dificuldades que demandam uma atenção maior e um trabalho dedicado a buscar soluções que favoreçam a aplicação dessa tecnologia no mundo e no Brasil. Em frente a sua evolução, nota-se que a IoT é uma grande promessa. Os equipamentos aos poucos estão se adaptando e com o advento da IPv6 virá a possibilidade de um avanço exponencial no desenvolvimento dos equipamentos para rede.

A IoT proporcionará a viabilidade de novos postos de trabalho para vários setores, principalmente, para a área de desenvolvimento de aplicativos voltados para os dispositivos conectados e na engenharia de telecomunicações, que precisará planejar a infraestrutura necessária para o pleno funcionamento da rede.

A segurança será um dos maiores desafios enfrentados, demandando uma pesquisa mais apurada em relação ao assunto e gerando uma necessidade latente de criação de ferramentas, conceitos ou equipamentos que consigam garantir a segurança das informações e dos dispositivos. Como exemplo de melhoria pode-se destacar a utilização de umacriptografia mais completa, assim como firewall, monitoramento, gerenciamento e acesso controlado, visando garantir a segurança de dentro para fora e vice-versa. Contudo, é importante dar um destaque especial ao hardware dos dispositivos, buscando aprimora-los a fim de garantir que eles não interfiram de forma negativa no desempenho geral.

Tratando-se do cenário brasileiro, as previsões de crescimento nesta área são otimistas, porém é importante ressaltar que algumas barreiras precisam ser ultrapassadas, como regulamentos, tributos, segurança e custos de operações. Portanto, é possível concluir que existe muito trabalho a ser realizado, visando inovar na criação de novos conceitos e tendências que viabilizem a implementação e expansão da IoT, diminuindo possíveis rejeições e receios e maximizando exponencialmente seus resultados e benefícios. 


\section{REFERÊNCIAS BIBLIOGRÁFICAS}

ASENSIO, Ángel; MARCO, Álvaro; BLASCO, Rubén; CASAS, Roberto. Protocol and Architecture to Bring Things into Internet of Things. Disponível em:<http://www.hindawi.com/journals/ijdsn/2014/158252/ >. Acesso em: maio 2020.

Atzori, L., Iera, A., \& Morabito, G. (2010). The internet of things: a survey. Computer Networks, 54(15), 2787-2805.

BASSI, A.; BAUER, M.; FIEDLER, M.; KRAMP, T.; VAN KRANENBURG, R.; Mattern, F., \& Floerkemeier, C. (2010). From the internet of computers to theinternet of things. Communications of the ACM, 6462, 242-259.

BRASIL. Ministério das Comunicações. Governo prepara plano para M2M e internet das coisas, 25 jun.2015. Disponível em: <http://www.comunicacoes.gov.br/sala-deimprensa/todas-as-noticias/institucionais/35836-governo-prepara-plano-para-m2m-e-internetdas-coisas>. Acesso em: maio 2020.

CHEN, Shanzhi; XU, Hui; LIU, Dake; HU, Bo; WANG, Hucheng. A Vision of IoT: Applications, Challenges, and Opportunities With China Perspective. IEEE Internet of Things Journal, VoL. 1, No. 4, August, 2014. Disponível em: <http://ieeexplore.ieee.org/stamp/stamp.jsp?arnumber=6851114> Acesso em: maio 2020.

COELHO, P. M. N. N. Rumo à Indústria 4.0. Coimbra, Portugal: Universidade de Coimbra: 2016. Disponível em: <https://estudogeral.uc.pt/handle/10316/36992> Acesso em maio de 2020.

DOMINGO, Mari. An overview of the Internet of Things for people with disabilities. Journal of Network and Computer Applications. Vol. 35, março, 2012.Páginas 584-596. Acesso em: maio 2020.

EXAME.com. Empresas apontam desafios para Internet das Coisas. Publicado em 08 de jan. 2014. Disponível em: <http://exame.abril.com.br/tecnologia/noticias/empresas-apontamdesafios-para-Internet-das-coisas >. Acesso em: maio 2020.

FERRAZ, Karen. Internet das Coisas: desafios que vão além da infraestrutura de redes. São Paulo, 2014. Disponível em: < http://itforum365.com.br/noticias/detalhe/114102/internetdascoisas-desafios-que-vao-alem-da-infraestrutura-das-redes>. Acesso em: maio 2020.

Fleisch, E. (2010). What is the internet of things? An economic perspective. Economics, management, and financial markets, 5(2), 125-157.

Gartner Inc. (2013). Gartner says the internet of things installed base will growto 26 billion units by 2020. Disponível em: <http://www.gartner.com/newsroom/id/2636073>. Acesso em: maio 2020.

GIL, A. C. Como elaborar projetos de pesquisa. São Paulo: Atlas, 1991.

GUBBI, Jayavardhana; BUYYA, Rajkumar; MARUSIC, Slaven; PALANISWAMI, Marimuthu. Internet of Things (IoT): A vision, architectural elements, and future directions. Future Generation Computer Systems. Volume 29, Issue 7, September 2013, Pages 1645-1660. Disponível em: 
<http://www.sciencedirect.com/science/article/pii/S0167739X13000241> Acesso em: maio 2020.

GUERRA, Rogério. Internet das Coisas: desafios que vão além da infraestrutura de redes Publicado por Karen Ferraz. Evento Futurecom 2014. São Paulo, 2014. Disponível em: $<$ http://itforum365.com.br/noticias/detalhe/114102/internet-das-coisas-desafios-que-vaoalemda-infraestrutura-das-redes $>$. Acesso em: maio 2020.

HERMANN, M.; PENTEK, T.; OTTO, B. Design Principles for Industrie 4.0 Scenarios: A Literature Review. Working paper, 2015.

ITU (2005). Internet reports — The internet of things. Disponível em: < https://www.itu.int/net/wsis/tunis/newsroom/stats/The-Internet-of-Things-2005.pdf >. Acesso em: maio 2020.

JARA, Antonio ; LADID, Latif; SKARMETA, Antonio. The Internet of Things through IPv6: An Analysis of Challenges, Solutions and Opportunities. Journal of Wireless Mobile Networks, Ubiquitous Computing, and Dependable Applications,2014. Disponível em: < http://iot6.eu/sites/default/files/imageblock/ipv6-iot6.pdf>. Acesso em: maio 2020.

Koreshoff, T. L., Robertson, T., \& Leong, T. W. (2013). Internet of things: Areview of literature and products. In Em Proceedings of the 25th Austra-lian Computer-Human Interaction Conference: Augmentation, Application,Innovation, Collaboration (335344). New York: ACM.

LAKATOS.E.M; MARCONI.M.A. Fundamentos de Metodologia Científica. São Paulo: $5^{\text {a }}$ ed., Atlas,2003.

LANGE, S.; MEISSNER, S. Enabling Thngs to talk: Designing IoT solutions with the IoT Architectural Reference Model. Ebook , Editora Springer Heidelberg New York Dordrecht London. Disponível em: <http://link.springer.com/book/10.1007\%2F978-3-642-40403-0〉. Acesso em: maio 2020.

LIU, Yi; WANG, He; WANG, Junyu; QIAN, Kan; KONG, Ning; WANG, Kaijiang; ZHENG, Lirong; SHI, Yiwei; ENGELS, Daniel. Enterprise-Oriented IoT Name Service for Agricultural Product Supply Chain Management. International Journal of Distributed Sensor Networks Volume 2015 (2015), Article ID 308165, 12 páginas. Disponível em: < http://www.hindawi.com/journals/ijdsn/2015/308165/>. Acesso em: maio 2020.

LOES, João; STEINHAUSER, Rafael. O que é a Internet das Coisas e como ela mudará suas vidas. Disponível em: <http://tecnologia.terra.com.br/o-que-e-a-Internet-das-coisas-ecomo-ela-mudara-a-suavida, 3e61c3b90c8ca410VgnVCM3000009af154d0RCRD.html>. Acesso em: maio de 2020.

MARÃO, Gabriel. Internet das Coisas: interoperabilidade é o nome do jogo. Disponível em:

$<$ http://convergenciadigital.uol.com.br/cgi/cgilua.exe/sys/start.htm?infoid=33841\&sid=15\&tp $\mathrm{l}=$ printerview $>$. Acesso em: maio 2020.

MARION, José Carlos; DIAS, Reinaldo; TRALDI, Maria Cristina. Monografia para cursos de administração, contabilidade e economia. São Paulo: Atlas, 2002. 
PERINI, Ricardo. IPv6: Novo protocolo chega para suprir o esgotamento de IP na América latina. Disponível em: http://www.profissionaisti.com.br/2014/11/ipv6novoprotocolo-chega-para-suprir-o-esgotamento-de-ip-na-america-latina/> Acesso em: maio de 2020.

SANNAPUREDDY, B. R. Pros \& Cons of Internet Of Things (IOT). Disponível em: $<$ https://www.linkedin.com/pulse/pros-cons-Internet-things-iot-bhaskarareddysannapureddy?trk=prof-post. $>$. Acesso em: maio de 2020.

SANTOS, Bruno P. et al. Internet das Coisas: da Teoria à Prática. 2017. Departamento de Ciências da Computação, UFMG. Disponível em:

<http://homepages.dcc.ufmg.br/ mmvieira/cc/papers/internet-das-coisas.pdf>. Acesso em: maio de 2020.

SCHWAB, K. A quarta revolução industrial. São Paulo: Edipro, 2016. 159 p.

SILVA, R. A importância da interoperabilidade. Disponível em:

<http://www.phpbrasil.com/artigo/-XVEH0RZGzGr/a-importancia-dainteroperabilidade> Acesso em maio de 2020.

SKARMETA, Antonio. Lightweight MIPv6 with IPSec Support. Mobile Information Systems. Vol. 10, 2014. Páginas 37-77. Acesso em: maio 2020.

SOLDATOS, John K. et al. Internet of Things Applications in Future Manufacturing. 2016. Disponível em:

<https://www.researchgate.net/publication/305033020_Internet_of_Things_Applications_in_ Future_Manufacturing>. Acesso em: maio de 2020.

SUGAYAMA, R.; NEGRELLI, E. Connected vehicle on the way of Industry 4.0. Paraná: Especialização Engenharia Automotiva, Universidade Tecnológica Federal do Paraná, 2015. Disponível em: <http://pdf.blucher.com.br.s3-sa-east1.amazonaws.com/engineeringproceedings/simea2016/PAP16.pdf $>$ Acesso em: maio de 2020 .

Tan, L., \& Wang, N. (2010). Future internet: the internet of things. In Anais da 3rdInternational Conference on Advanced Computer Theory and Engineering.Chengdu, China: ICACTE.

TRAVASSOS, Gustavo. Internet das Coisas: expectativas e desafios para 2015. São Paulo, 2015. Disponível em: <http://startupi.com.br/2015/01/internet-das-coisas-expectativasedesafios- em-2015/>. Acesso em: maio 2020.

ZAMBARDA, Pedro. 'Internet das Coisas': entenda o conceito e o que muda com a tecnologia. Disponível em: <http://www.techtudo.com.br/noticias/noticia/2014/08/Internetdas-coisas-entenda-oconceito-e-o-que-muda-com-tecnologia.html >. Acesso em: maio de 2020.

ZASLAVSKY, A.; PERERA, C. Context Aware Computing for The Internet of Things: A Survey. Disponível em: < http://arxiv.org/pdf/1305.0982.pdf>. Acesso em maio de 2020. 